\title{
Controladores del Tiempo y el Envejecimiento: Núcleo Supraquiasmático y Glándula Pineal
}

\author{
Aging Process Entrainment: Suprachiasmatic Nucleus and Pineal Gland \\ Parménides Guadarrama-Ortiz*; Ricardo Ramírez-Aguilar*; Alejandro Madrid-Sánchez; \\ Carlos Castillo-Rangel ${ }^{* * *}$; Diana Carrasco-Alcántara ${ }^{* * * *}$ \& Raúl Aguilar-Roblero*****
}

GUADARRAMA-ORTIZ, P.; RAMÍREZ-AGUILAR, R.; MADRID-SÁNCHEZ, A.; CASTILLO-RANGEL, C.; CARRASCOALCÁNTARA, D. \& AGUILAR-ROBLERO, R. Controladores del tiempo y el envejecimiento: núcleo supraquiasmático y glándula pineal. Int. J. Morphol., 32(2):409-414, 2014.

RESUMEN: El núcleo supraquiasmático (NSQ) es el principal reloj biológico de los mamíferos y sincroniza la actividad de la glándula pineal al ciclo luz-oscuridad a través de una vía polisináptica. El efecto de asa de retroalimentación neuroendocrina se lleva a cabo por la melatonina. El presente trabajo pretende demostrar que la glándula pineal modula la sensibilidad a la luz en el NSQ. Se utilizaron ratas Wistar, y se asignaron a 3 grupos: grupo A (falsa pinealectomía -sham-, sin luz), grupo B (falsa pinealectomía -sham- + luz) y grupo $\mathrm{C}$ al cual se le realizó la pinealectomía + luz, después de la manipulación se sacrifican para realizar inmunohistoquímica para c-Fos y al final conteo celular por técnica de estereología. Se obtuvo una reducción del 46,8\% del promedio de células inmunorreactivas a c-Fos en el grupo C en comparación del grupo B. Este trabajo muestra que la sensibilidad a la luz está modulada por la actividad de la glándula pineal.

PALABRAS CLAVE: Pinealectomía; c-Fos; Falsa pinealectomía; Núcleo supraquiasmático.

\section{INTRODUCCIÓN}

Los ritmos biológicos, son una característica fundamental de los seres vivos, y de particular importancia son los ciclos cercanos a las 24 horas, llamados ritmos circadianos (lat. circa: cercano, diem: día) (Saper et al., 2005). En los organismos multicelulares, todos los procesos fisiológicos muestran oscilaciones a lo largo del día. A estas oscilaciones se les conoce como oscilaciones circadianas y son generadas y moduladas por el núcleo supraquiasmático (NSQ) (Aguilar-Roblero et al., 1998). El núcleo supraquiasmático (NSQ) se encuentra en la parte anterior del hipotálamo (Saper et al.; Moore \& Eichler, 1972; Moore \& Lenn, 1972) (Fig. 1A). El tercer ventrículo divide al par de núcleos supraquiasmáticos, medialmente (Moore \& Lenn) (Fig. 1B-C); se divide de una forma gruesa en dos regiones, una dorsomedial (dm), y la otra ventrolateral (vl). La región dorsomedial contiene células pequeñas, con cadenas neuronales con oposición soma-soma y es la principal zona de eferencias del NSQ (Swanson \& Cowan, 1975) y la re- gión ventrolateral principalmente es la zona retinorrecipiente de las aferencias del Tracto retinohipotalámico (TRH) (Hendrickson et al., 1972) (Fig. 1).

La aferencia mas estudiada es la que se origina en la retina y forma el tracto $\mathrm{TRH}$ a partir de las células ganglionares de la retina (Klein \& Moore, 1979). Se sabe que las proyecciones de TRH son suficientes para mantener la sincronización del NSQ a la luz, ya que lesiones de este tracto impiden que el NSQ ajuste su fase a ciclos de luz. Las vías que forman este tracto son independientes de las vías clásicas que participan en la percepción visual.

Una de las eferencias del NSQ mas importantes y que a su vez controla la secreción de melatonina, es la vía multisináptica a la glándula pineal (Klein \& Moore; PerreauLenz et al., 2003; Korf et al., 2003); Del NSQ, la proyección a la porción parvicelular dorsal del núcleo

* Residente de Neurocirugía, Hospital Regional $1^{\circ}$ de Octubre, ISSSTE, México D.F., México.

** Residente de Neurocirugía, Hospital Juárez de México, SSA, México D.F., México.

**** Profesor titular del Curso de Neurocirugía, Universidad Nacional Autónoma de México, Hospital Regional $1^{\circ}$ de Octubre, ISSSTE; Director de la Clínica de Neurotraumatología S. C., México D.F., México.

***** Residente de la Dirección General Adjunta de Epidemiología, SSA, México D.F., México.

****** Investigador Titular C, División de Neurociencias, Instituto de Fisiología Celular, Universidad Nacional Autónoma de México, México D.F., 04510, México. 
paraventricular del hipotálamo (PVN) (Lu et al., 2001) activa neuronas que proyectan sus axones a las neuronas simpáticas preganglionares de la columna intermediolateral (CIL) del cordón espinal y neuronas simpáticas noradrenérgicas del ganglio cervical superior (GCS) que forman los axones de nervus conarii, los cuales cursan intracranealmente a los lados de la tienda del cerebelo y se fusionan en la línea media para penetrar en la glándula por su polo distal posterior (Drijfhout et al., 1996). Y de esta manera el NSQ se comunica con la glándula pineal, siendo esta vía el mayor mecanismo de regulación del ciclo de melatonina (Fig. 2).

La Glándula pineal como regulador neuroendocrino: las fibras simpáticas que inervan esta glándula forman 2 grupos principales a saber: a) fascículos pequeños que penetran la cápsula junto con los vasos sanguíneos, y b) el nervus conarii. La glándula pineal, está rodeada por una capa pial que la suspende en el líquido cefalorraquídeo, en el receso de la pineal, directamente debajo del esplenio del cuerpo calloso y por encima de la lámina cuadrigémina (Fig. 3).

La melatonina es liberada siguiendo un ritmo circadiano con altos niveles en la noche y niveles bajos en el día. La liberación de noradrenalina dentro de la glándula pineal durante la noche estimula la síntesis de melatonina (Drijfhout et al.). La activación de receptores b1 adrenérgicos causa un incremento en la expresión del gen que codifica para la arilalquilamina-N-acetiltransferasa (AA-NAT), este proceso inmediatamente produce una aumento en la síntesis y secreción de melatonina (Roseboom et al., 1996). En ratas, el acido $\gamma$-aminobutírico (GABA) liberado en el PVN está involucrado en la inhibición de la síntesis de melatonina inducida por luz (Kalsbeek et al., 2000), lo cual sugiere que
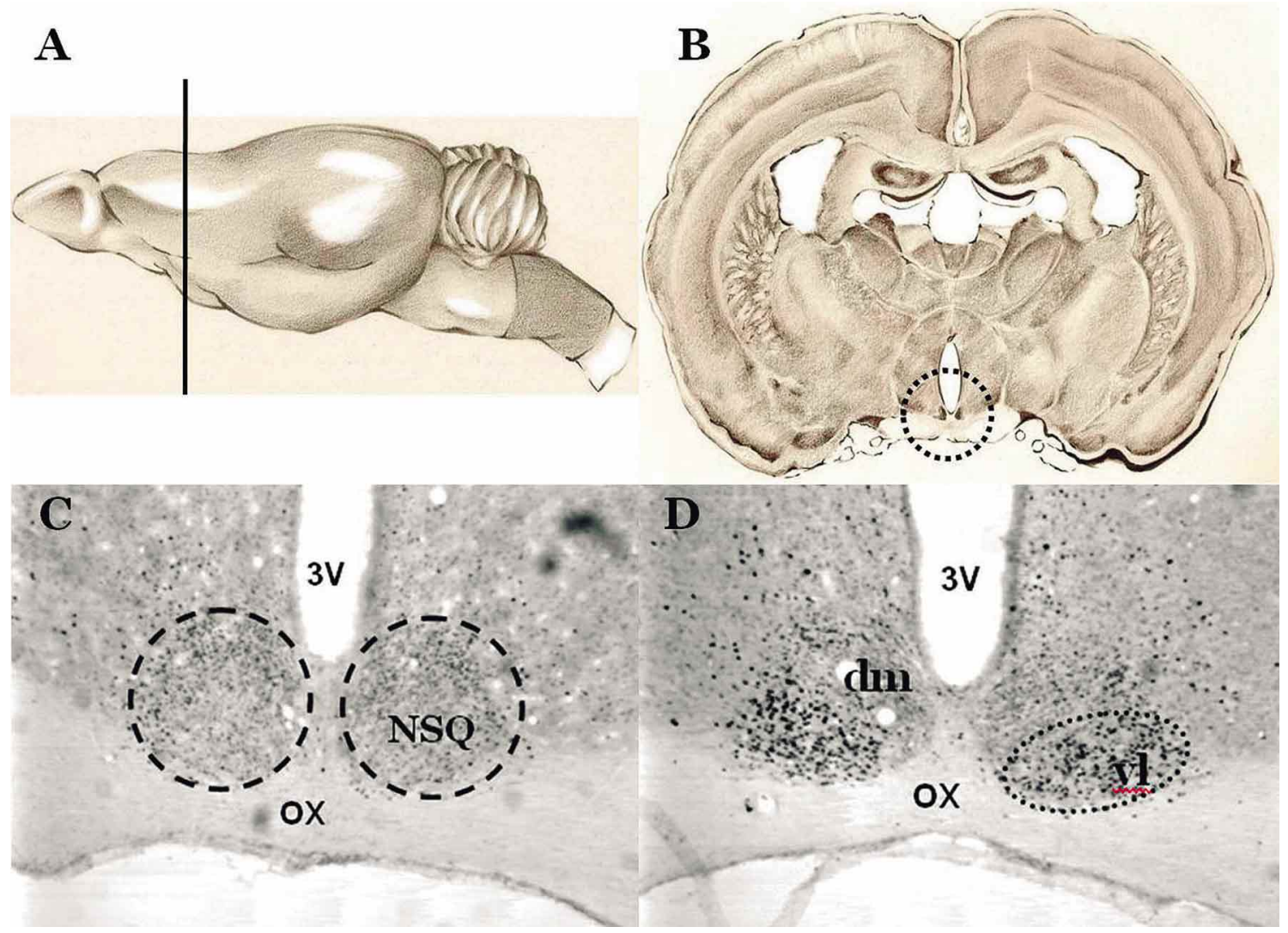

Fig. 1. Dibujos de cerebro de rata y con corte coronal del mismo y fotomicrografía con marcaje de células inmunorreactivas para c-fos, que localizan y describen anatómicamente al NSQ. A.- Dibujo de una vista lateral del cerebro de rata, la línea presenta el sitio de corte, en región del hipotálamo anterior. B.- Dibujo que representa una rebanada en corte coronal donde se aprecia en un circulo con línea discontinua, la localizacion del NSQ y su relación con el quiasma óptico (ox). C.Fotografía del NSQ, que se localiza por una alta densidad celular inmediatamente superior al ox, dentro de una línea discontinua y ambas estructuras divididas por el tercer ventrículo (3V). D.- Regiones celulares intranucleares bien delimitadas, la línea punteada muestra la región ventrolateral (vl) -retinorecipiente al tracto retinohipotalámico- y la región dorsomedial (dm). (Preparación realizada por el autor en el laboratorio y bajo la dirección del Dr. Raúl Aguilar Roblero, Departamento de Neurociencias, Instituto de Fisiología, UNAM; Dibujos superiores, técnica lápiz, propiedad del autor). 


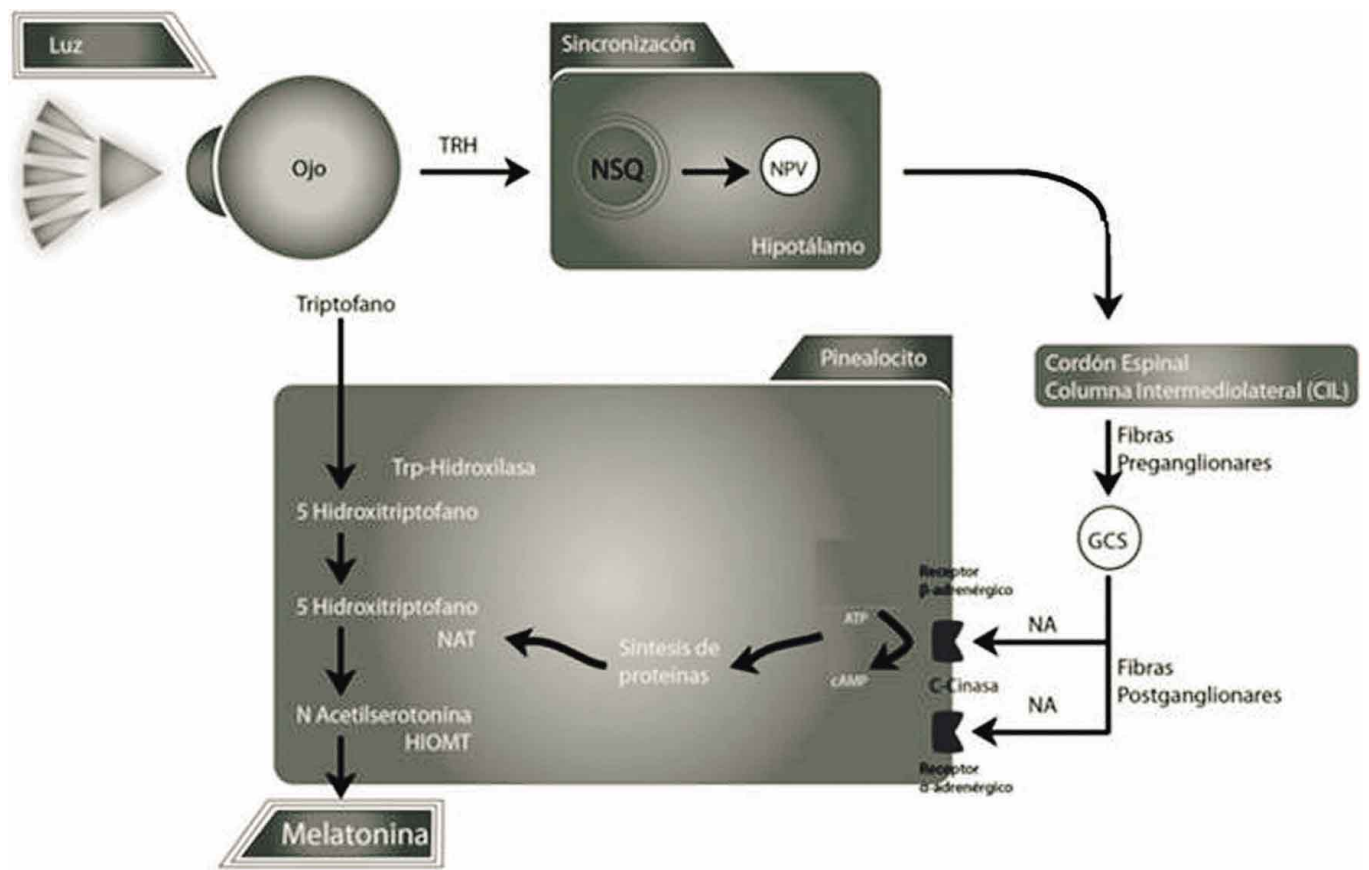

Fig. 2. Vía eferente, núcleo supraquiasmático-glándula pineal. Se muestra la vía de entrada de la luz y la comunicación del NSQ a la glándula pineal por medio de una vía multisináptica. Después del relevo por el ganglio cervical superior (GCS), los axones llegan a la glándula pineal (como se describe en el texto) y estimulan receptores adrenérgicos, los cuales a su vez desencadenan la activación de segundos mensajeros y activan la N-acetil-transferasa, enzima limitante en la formación de melatonina. TRH= tracto retinohipotalámico, $\mathrm{NSQ}=$ núcleo supraquiasmático; $\mathrm{NPV}=$ núcleo paraventricular del hipotálamo; $\mathrm{CIL}=$ columna intermediolateral, $\mathrm{GCS}=$ ganglio cervical superior, NA= noradrenalina, NAT $=\mathrm{N}$-acetil-transferasa, $\mathrm{HIOMT}=$ hidroxiindol-Ometiltransferasa.

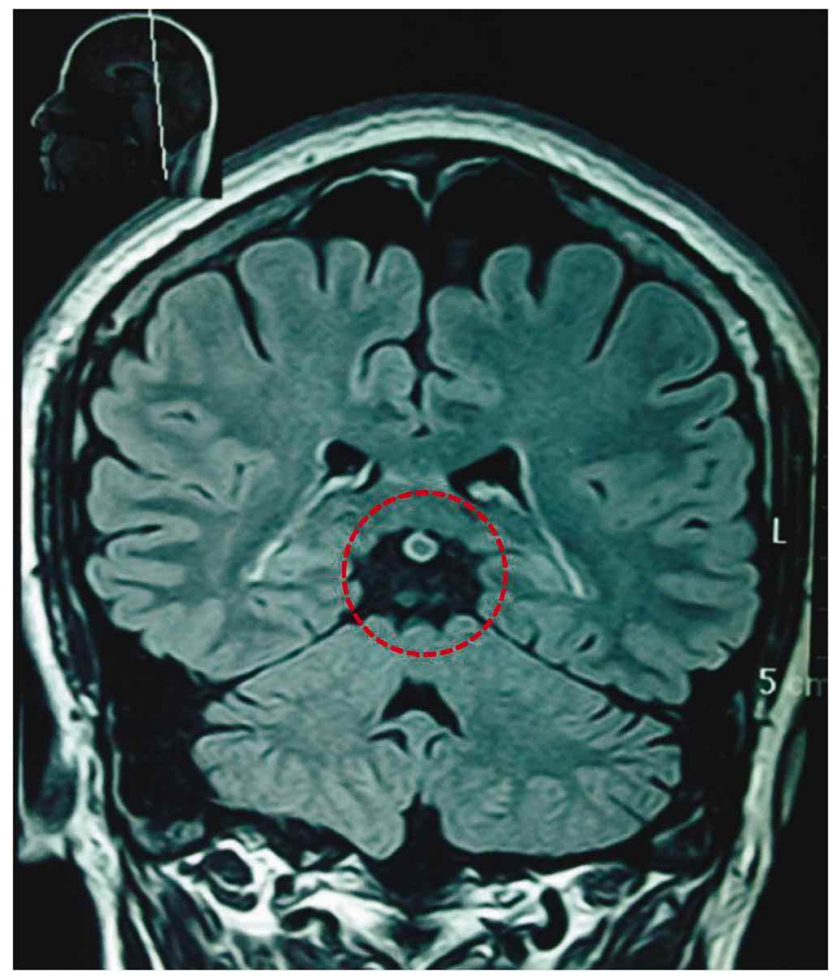

el NSQ controla la síntesis de melatonina por imposición de una señal inhibitoria gabaérgica sobre la vía PVN-pineal durante el día. Los receptores a la melatonina se encuentran en sistema nervioso central y tejido periférico; en sistema nervioso central principalmente en tres sitios: núcleo supraquiasmático, núcleo paraventricular del tálamo, pars tuberalis (PT) (Ross \& Morgan, 2002) sitios diencefálicos para el control de la ritmicidad circadiana y neuroendocrina y tejidos periféricos (retina, hígado, linfocitos y plaquetas) (Sack et al., 2000).

Así la presencia de receptores de melatonina en el NSQ indican la existencia de una asa de retroalimentación neuroendocrina dentro del sistema circádico, la cual puede complementar el ajuste de fase que lleva a cabo el tracto

Fig. 3. Corte coronal de resonancia magnética de encéfalo, pictograma en la parte superior donde se muestra el sitio de corte; la línea discontinua, muestra la glándula pineal y su relación con otras estructuras, especialmente con la lámina cuadrigémina la cual se observa en la parte inferior del círculo rojo (Imagen tomada en Clínica de Neurotraumatología S.C.). 
retinohipotalámico.En algunos vertebrados inferiores la pineal funciona como un oscilador circadiano, sin embargo su papel en los mamíferos no es claro.

La inmunorreactividad en las neuronas donde se activan genes de expresión temprana del tipo de c-Fos son un índice muy confiable de la actividad neuronal, tanto espontánea, como en respuesta a la actividad transináptica, y en el caso muy particular del NSQ tiene una relevancia en cuanto a los mecanismos de adelantos y retrasos de fase del reloj circadiano en la curva de respuesta de fase (CRF) (Reiter et al., 1991).

El presente trabajo pretende evaluar si la glándula pineal, a través de la liberación de melatonina, es capaz de modular la sensibilidad a la luz en el NSQ y la pinealectomía provocará una disminución en la fotoinducción de c-Fos en el NSQ. En particular, aún se desconocen los mecanismos precisos por los cuales la luz y la melatonina, modulan la excitabilidad de las neuronas en el NSQ, esto podría ser a través de genes reloj, el calcio intracelular, la melatonina, y la acetilcolina (von Gall et al., 2005).

\section{MATERIAL Y MÉTODO}

Se realizó un estudio experimental en ratas en el Instituto de Fisiología Celular, Departamento de Neurociencias, UNAM. Se utilizaron 60 ratas macho, cepa Wistar, de $200 \mathrm{~g}$, de los cuales se asignaron tres grupos: Grupo A, control, al que se le realizó la craniectomía con apertura de la duramadre sin extracción de la pineal (falsa pinealectomía= sham), al final sin dar pulso de luz (sham, sin luz); Grupo B, control, al que se les realizó la craniectomía con apertura de la duramadre sin extracción de la pineal (sham) y si estuvieron expuestos a pulso de luz (sham+luz); Grupo C, al que se les extrajo la glándula pineal mediante una craniectomía perietoocipital y se expuso a pulso de luz (Pinealectomizados (Px)): Px+luz). Posterior a este procedimiento se dejaron en recuperación 10 días en obscuridad continua, y mediante el registro continuo de actividad locomotora o ingesta de agua se obtuvo la expresión endógena del ritmo circadiano, y con esto se determinó la hora a la que se dará un pulso de luz (800 lux), la hora es determinada con la curva de respuesta de fase (CRF), usando solo la hora circádica 12 (circadian time 12=CT12). Al terminar los animales se sacrificaron y se preservó la muestra cerebral, con la que se realizó la técnica de inmunohistoquímica para c-Fos. Al final se contaron las células mediante la técnica de estereología.

\section{RESULTADOS}

Con el fin de comprobar la hipótesis estudiamos el efecto de la pinealectomía sobre la fotoinducción de c-Fos en el NSQ a la hora circádica CT12. En el grupo B (sham + luz), los pulsos de luz aplicados indujeron una robusta expresión de c-Fos en las neuronas del núcleo supraquiasmático, esto comparado con el grupo A (sham + sin luz), mientras que los pulsos de luz aplicados en el grupo $\mathrm{C}$ (pinealectomizados + luz) presentaron una reducción en el promedio del número de neuronas que expresaban c-Fos en CT12 en comparación con el grupo B (sham + luz) (Fig. 4). Posteriormente mostramos la cuantificación estereológica donde se puede observar que comparando el grupo $\mathrm{B}$ con el grupo $\mathrm{C}$ hubo una menor expresión de las células inmunorreactivas a c-Fos (Fig. 5). En el

\section{CT 12}

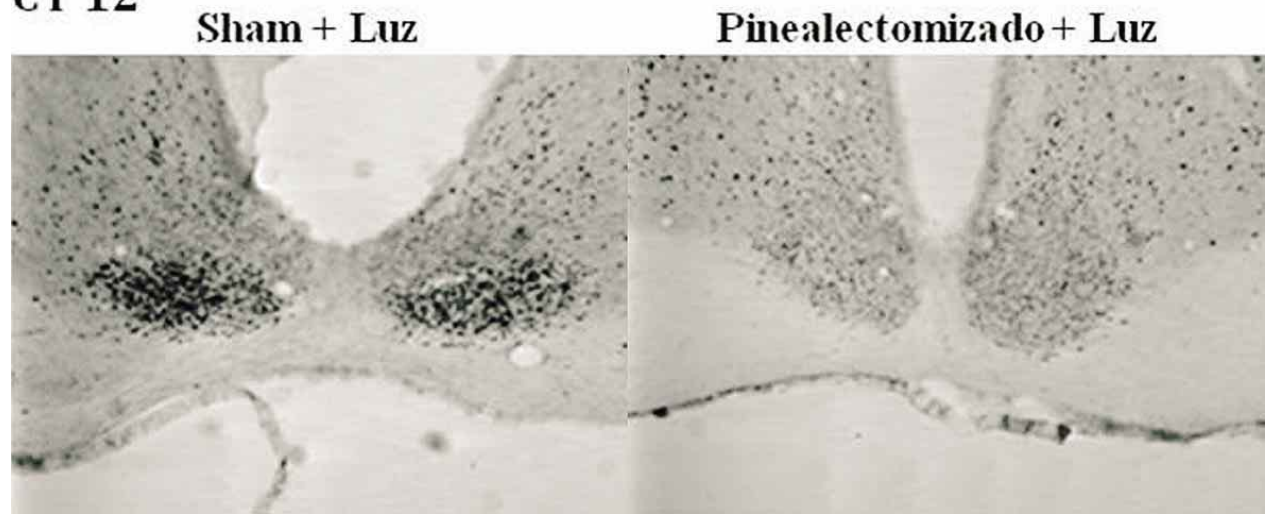

Fig. 4. Cortes coronales comparativos del NSQ que muestran células inmunorreactivas a c-Fos en sujetos Sham + luz y Px + luz. Se puede observar una notable fotoinducción de c-Fos en el NSQ en CT12 de Sham + luz respecto a pinealectomizados + luz. CT= circadian time, hora circadica (Resultados obtenidos por el autor en el laboratorio de Dr. Raúl Aguilar, Instituto de Fisiología Celular, Departamento de Neurociencias, UNAM). 
Cuadro 1 se observa el porcentaje del promedio de células inmunorreactivas a c-Fos por intervención aplicada en cada uno de los grupos; en el grupo A, un $5 \%$ (IC 0,05-0,06), grupo B, un $64 \%$ (IC 0,62-0,65) y en el grupo C, un $30 \%$ (IC 0,28-0,31) mostraron inmunorreactividad. Se obtuvo una reducción del 46,8\% del promedio de células inmunorreactivas a c-Fos en el grupo $\mathrm{C}$ en comparación del grupo B.

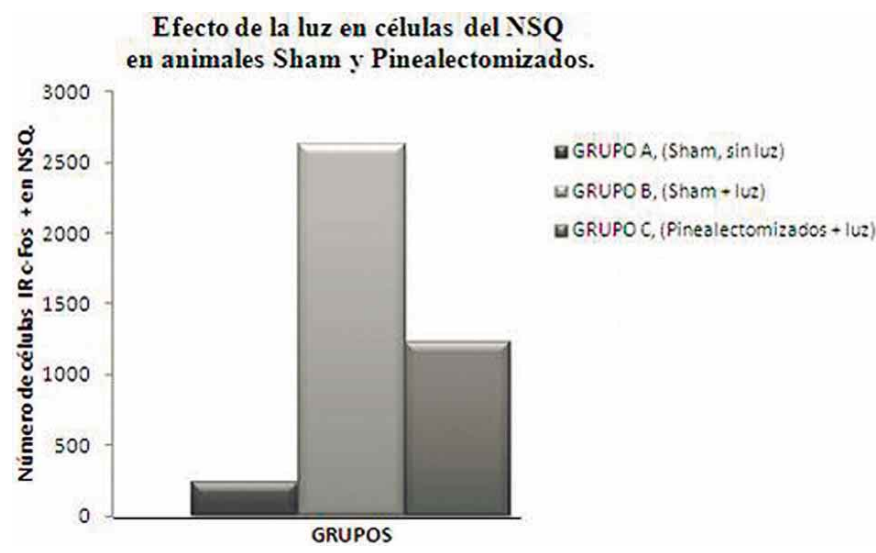

Fig. 5. Muestra la comparación del promedio del número de células inmunoreactivas a c-Fos de los grupos de estudio (Resultados obtenidos por el autor en el laboratorio de Dr. Raúl Aguilar, Departamento de Neurociencias, Instituto de Fisiología, UNAM).

\section{DISCUSIÓN}

Estos resultados indican que la pinealectomía disminuye la sensibilidad del núcleo supraquiasmático a la luz y dicho efecto podría estar a nivel de la retina o directamente en las neuronas del núcleo supraquismático. Los resultados de los experimentos de fotoinducción de c-Fos, indican que la pineal participa en el mecanismo de sincronización a la luz del sistema circádico de los mamíferos. Dicha participación consistiría en primera instancia en modular la sensibilidad del supraquiasmático a la luz ya sea directa o indirectamente. De tal forma que durante la oscuridad, cuando la melatonina se secreta, las neuronas del NSQ son más sensibles a la luz. Además, es posible que la inhibición de la secreción de melatonina durante la aplicación de los pulsos de luz también facilite la respuesta de las neuronas del supraquiasmático a la estimulación de la vía retinohipotalámica, así la melatonina modula la actividad de las neuronas del NSQ. Actualmente estamos realizando experimentos orientados a determinar si la pinealectomía afecta la expresión funcional de receptores a glutamato en especial los de $\mathrm{N}$-metil D-aspartato (NMDA) en el supraquiasmático, ya que éstos son los receptores involucrados en la transmisión sináptica entre las terminales de la vía retinohipotalámica y las neuronas del núcleo supraquiasmático y se elucidará si el efecto es solo de recetores en la membrana o también es de manera directa en el núcleo celular en los genes reloj.

\section{CONCLUSIONES}

Al igual que en otros vertebrados como los peces, anfibios, reptiles y aves, en los mamíferos la glándula pineal es un componente muy importante del sistema circádico que junto con el núcleo supraquiasmático forman un asa de retroalimentación neuroendócrina. Esta asa participa principalmente en la sincronización del supraquiasmático a la luz y en el acoplamiento de diversos osciladores circadianos. En la sincronización a la luz, la glándula pineal participa como modulador de la sensibilidad del supraquiasmático a los estímulos que codifica la iluminación ambiental, la inhibición de la secreción de melatonina por la exposición a pulsos de luz permite la adecuada transmisión en la vía retinohipotalámica. En el acoplamiento la melatonina y otras hormonas producidas por la pineal, trasmiten la fase de ciclo circadiano a todo el organismo que en caso particular de los osciladores circadianos ubicados en el supraquiasmático les permiten funcionar como un reloj circadiano coherente.

Este trabajo contribuye en una mejor comprensión de ciertas enfermedades, ya que se sabe que aproximadamente el $50 \%$ de los niños con autismo muestran alteraciones en los patrones del sueño, y a su vez se relaciona con las concentraciones de melatonina disminuidas.

En los pacientes con Enfermedad de Alzheimer existe una severa alteración en el ciclo sueño-vigila, y algunos presentan agitación nocturna que se caracteriza por una desorganización circadiana, así como una capacidad reducida de mantener la atención sobre estímulos externos, desorganización en el pensamiento y en la habla, alteraciones motoras que incluyen movimientos repetidos y modificaciones en la percepción y emoción. En este grupo de pacientes de igual manera se presentan una disminución en la secreción de melatonina en el líquido cerebroespinal y algunos autores sugieren que las alteraciones del sueño son resultado de la pérdida en las conexiones de la pineal con el núcleo supraquiasmático, lo que modifica el ritmo de síntesis y secreción de la melatonina.

La cefalea hípnica (primaria asociada con el sueño) probablemente sea un trastorno cronobiológico. La pérdida de las neuronas del NSQ que ocurre durante en envejecimiento permitiría explicar la mayor prevalencia de cefalea hípnica en sujetos de edad relativamente avanzada y su respuesta a la terapia de melatonina.

AGRADECIMIENTOS. Parcialmente financiado por el proyecto IN-204811 del PAPIIT/DGAPA/UNAM y el proyecto 128528 de CONACYT a RAR. 
GUADARRAMA-ORTIZ, P.; RAMÍREZ-AGUILAR, R.; MADRID-SÁNCHEZ, A.; CASTILLO-RANGEL, C.; CARRASCOALCÁNTARA, D. \& AGUILAR-ROBLERO, R. Aging process entrainment: suprachiasmatic nucleus and pineal gland. Int. J. Morphol., 32(2):409-414, 2014.

SUMMARY: The suprachiasmatic nucleus (SCN) is the main and major biological clock in mammals and is responsible for the synchronization of the pineal gland to the light/darkness cycle through a polysynaptic pathway. The neuroendocrine feedback loop effect is carried out by melatonin. This study was carried out to demonstrate that the pineal gland adjusts the sensibility to light in the suprachiasmatic nucleus. Wistar rats were allocated in 3 groups: Group A (sham pinalectomy, without light), group B (sham pinealectomy + light) and group C which underwent real pinalectomy + light. After the intervention the animals were slain to perform immunohistochemistry for c-Fos and cell counting by stereology technique. A $46.8 \%$ average reduction in c-Fos immunoreactive cells was achieved in-group C as compared with group B. The present work shows that sensibility to the light is modulate by the activity of the pineal gland.

KEY WORDS: Pinealectomy; c-Fos; Sham operation; Suprachiasmatic nucleus.

\section{REFERENCIAS BIBLIOGRÁFICAS}

Aguilar-Roblero, R.; Escandón-Calderón, J.; Salazar-Juárez, A.; Caldelas, I.; Granados-Fuentes, D. \& Escobar, C. Phase relations between host and grafted scn depend on graft location in rats. Biol. Rhythm Res., 29(5):521-9, 1998.

Drijfhout, W. J.; van der Linde, A. G.; Kooi, S. E.; Grol, C. J. \& Westerink, B. H. Norepinephrine release in the rat pineal gland: the input from the biological clock measured by in vivo microdialysis. J. Neurochem., 66(2):748-55, 1996.

Hendrickson, A. E.; Wagoner, N. \& Cowan, W. M. An autoradiographic and electron microscopic study of retinohypothalamic connections. Z. Zellforsch. Mikrosk. Anat., 135(1): 1-26, 1972.

Kalsbeek, A.; Garidou, M. L.; Palm, I. F.; Van Der Vliet, J.; Simonneaux, V.; Pévet, P. \& Buijs, R. M. Melatonin sees the light: blocking GABA-ergic transmission in the paraventricular nucleus induces daytime secretion of melatonin. Eur. J. Neurosci., 12(9):3146-54, 2000.

Klein, D. C. \& Moore, R. Y. Pineal N-acetyltransferase and hydroxyindole-O-methyltransferase: control by the retinohypothalamic tract and the suprachiasmatic nucleus. Brain Res., 174(2):245-62, 1979.

Korf, H. W.; Von Gall, C. \& Stehle, J. The circadian system and melatonin: lessons from rats and mice. Chronobiol. Int., 20(4):697-710, 2003.

Lu, J.; Zhang, Y. H.; Chou, T. C.; Gaus, S. E.; Elmquist, J. K.; Shiromani, P. \& Saper, C. B. Contrasting effects of ibotenate lesions of the paraventricular nucleus and subparaventricular zone on sleep-wake cycle and temperature regulation. J. Neurosci., 21(13):4864-74, 2001

Moore, R. Y. \& Eichler, V. B. Loss of a circadian adrenal corticosterone rhythm following suprachiasmatic lesions in the rat. Brain Res., 42(1):201-6, 1972.

Moore, R. Y. \& Lenn, N. J. A retinohypothalamic projection in the rat. J. Comp. Neurol., 146(1):1-14, 1972.
Perreau-Lenz, S.; Kalsbeek, A.; Garidou, M. L.; Wortel, J.; van der Vliet, J.; van Heijningen, C.; Simonneaux, V.; Pévet, P. \& Buijs, R. M. Suprachiasmatic control of melatonin synthesis in rats: inhibitory and stimulatory mechanisms. Eur. J. Neurosci., 17(2): 221-8, 2003.

Reiter, R. J. Melatonin synthesis: multiplicity of regulation. Adv. Exp. Med. Biol., 294:149-58, 1991.

Roseboom, P. H.; Coon, S. L.; Baler, R.; McCune, S. K.; Weller, J. L. \& , D. C. Melatonin synthesis: analysis of the more than 150 -fold nocturnal increase in serotonin $\mathrm{N}$-acetyltransferase messenger ribonucleic acid in the rat pineal gland. Endocrinology, 137(7):303345, 1996.

Ross, A. W. \& Morgan, P. J. The pars tuberalis as a target of the central clock. Cell Tissue Res., 309(1):163-71, 2002.

Sack, R. L.; Brandes, R. W.; Kendall, A. R. \& Lewy, A. J. Entrainment of free-running circadian rhythms by melatonin in blind people. $N$. Engl. J. Med., 343(15):1070-7, 2000.

Saper, C. B.; Lu, J.; Chou, T. C. \& Gooley, J. The hypothalamic integrator for circadian rhytms. Trends Neurosci., 28(3):152-7, 2005.

Swanson, L. W. \& Cowan, W. M. The efferent connections of the suprachiasmatic nucleus of the hypothalamus. J. Comp. Neurol., 160(1):1-12, 1975.

von Gall, C.; Garabette, M. L.; Kell, C. A.; Frenzel, S.; Dehghani, F.; Schumm-Draeger, P. M.; Weaver, D. R.; Korf, H. W.; Hastings, M. H. \& Stehle, J. H. Rhythmic gene expression in pituitary depends on heterologous sensitization by the neurohormone melatonin. Nat. Neurosci., 5(3):234-8, 2005.

\section{Dirección para Correspondencia: \\ Dr. Parménides Guadarrama-Ortiz \\ Av. Politécnico Nacional No. 1669. \\ Col. Magdalena de la Salinas \\ Deleg. Gustavo A. Madero \\ México D.F. 07300 \\ MÉXICO}

Email: dvorak50@hotmail.com

Recibido : 25-11-2013

Aceptado: 21-02-2014 